NOTICE: this is the author's version of a work that was accepted for publication in Journal of sound and vibration. Changes resulting from the publishing process, such as peer review, editing, corrections, structural formatting, and other quality control mechanisms may not be reflected in this document. Changes may have been made to this work since it was submitted for publication. A definitive version was subsequently published in Journal of sound and vibration, [VOL 332, ISSUE 21, (10/2013)] DOI 10.1016/j.jsv.2013.05.012

\title{
Numerical estimation of coupling loss factors in building acoustics
}

\author{
C. Díaz-Cereceda*1 ${ }^{* 1}$ J. Poblet-Puig ${ }^{\dagger 1}$ and A. Rodríguez-Ferran ${ }^{\ddagger 1}$ \\ ${ }^{1}$ Laboratori de Càlcul Numèric, E.T.S. d'Enginyers de Camins, Canals \\ i Ports de Barcelona, Universitat Politècnica de Catalunya
}

\begin{abstract}
A study on the optimal procedure for obtaining SEA (statistical energy analysis) coupling loss factors (CLF) numerically is presented. The energies of a SEA system with two subsystems (one excited, the other one unexcited) are obtained from deterministic numerical simulations. Three different ways of isolating the CLF are explored: from the power balance of the excited subsystem (first approach) or the unexcited subsystem (second approach) and from the power transmitted through the connection (third approach). An error propagation analysis shows that the first approach is unreliable and that the second approach is the best option. As application examples, the CLF between some typical building structures is computed. These examples illustrate the potential of the estimated CLFs to solve larger problems with SEA and show the influence of the type of excitation on the coupling loss factor estimation. Finally, a simplified technique to account for the effect of studs in double walls with SEA is presented.
\end{abstract}

Keywords: Statistical energy analysis, error propagation, coupling loss factor, numerical simulation

\section{Introduction}

Dealing with real-life vibroacoustic problems with statistical energy analysis (SEA) is not a straightforward task. The obtention of the coupling loss factors (CLF) may be a limiting factor for complex connections. In this paper a study on the optimal technique for obtaining the coupling loss factors between two subsystems, independently of the device connecting them, is presented.

\footnotetext{
*e-mail:cristina.diaz.cereceda@gmail.com

${ }^{\dagger}$ correspondence: UPC, Campus Nord B1, Jordi Girona 1, E-08034 Barcelona, Spain, e-mail: jordi.poblet@upc.edu

‡e-mail: antonio.rodriguez-ferran@upc.edu
} 
Statistical energy analysis is an energy-based approach to vibroacoustic problems, widely used in building design due to its low computational cost and simplicity. It was first described by Lyon [17] as a framework of analysis based on the sound behaviour at high frequencies, and consists in performing power balances in an averaged way between the different subsystems of the vibroacoustic system. Due to this averaged nature, SEA is not designed to take into account small details of the problem geometry or heterogeneities.

For complex systems, the parameters required in the SEA formulation, such as the coupling loss factors, cannot be calculated analytically. One option for obtaining these parameters is to identify them from laboratory measurements. Some authors working in that direction are Semprini and Barbaresi [26], De Langhe and Sas [16], Gélat and Lalor [12], Renji and Mahalakshmi [25] or Bies and Hamid [1]. Most of them use the power injection method (PIM). This is a very common technique for estimating coupling loss factors from experiments. It is based on measuring energies and powers experimentally and fitting the CLFs from power balances.

Another option to estimate these parameters is using deterministic formulations and the help of numerical methods. In the past decades, several authors have presented different approaches to estimate CLFs from numerical simulations. Some of them [10, 29, 28, 33] use finite element methods (FEM) to obtain the energy fluxes in the problem, and then estimate SEA parameters from them. They mainly focus on mechanical problems with no acoustic interaction and do not apply the obtained parameters for solving larger problems.

Maxit and Guyader [21] estimate the coupling loss factors from modal parameters of the SEA subsystems. They use modal analysis to obtain these parameters by means of simplifying the problem with the help of substructuring. The substructuring criterion restricts the approach to domains where there are two distinct subsystems and one of them has a clearly stiffer behaviour than the other one. Problems where the subsystems are not in direct contact but connected by a third element, such as double walls, should be approached differently. Maxit and Guyader apply their approach to obtain coupling loss factors between beams and plates with common edges and Totaro et al. [31] use the same approach to compute coupling loss factors between structures and cavities.

Finally, Thite and Mace [30] deal with the idea of obtaining robust estimators of these parameters from the deterministic results. They explore the usefulness of Montecarlo simulations for obtaining robust enough values of the coupling loss factors to be used later in different types of problems.

The main contribution of this paper is the study on the optimal approach for obtaining the coupling loss factors between two subsystems from numerical simulations. The choice of the approach is based on the error propagation committed by operating the approximated values of the energies. Other contributions derived from exploiting the chosen technique are the following:

- An analytical expression, based on the wave approach, for the coupling loss factor between two plates connected by an elastic rotational joint.

- An analysis, based on numerical simulations, of the effects of considering the cavity between the two leaves of a double wall as an SEA subsystem or as a 
connection between subsystems. It is followed by the proposal of a combined approach that accounts for all the transmission phenomena relevant in the double wall.

- An analysis on the influence of the excitation used in the CLF estimation when this factor is applied for solving larger vibroacoustic problems with SEA.

- A simplified approach to obtain the coupling loss factor caused by the studs between the two leaves of a double wall. Also a comparison of the coupling effect of the air cavity and the effect of the studs.

An outline of the paper follows. The bases of the CLF estimation are explained in Section 2. Three different ways of computing the CLF once the energies of the subsystems are known are described. An error propagation analysis is performed, identifying the best expressions to use in the CLF computation. At the end of the section, the deterministic approach [8, 9] is briefly reviewed. In Section 3 some application examples are shown. The coupling loss factor between structures connected by different mechanical devices is obtained and compared with analytical expressions in order to validate the technique. The computed values are then used to solve more complex problems with low computational cost. The coupling loss factors associated to double walls are also computed. The effect of the air cavity is analysed and compared with typical SEA expressions. Moreover, an example of the applicability of the CLF estimations to obtain the sound reduction index of double walls is shown, considering walls with and without studs between the leaves. Finally, the conclusions of the work are presented in Section 4.

\section{Methodology}

The technique for computing the CLF between two subsystems is presented here. For any connecting device between them, the deterministic vibroacoustic problem is solved numerically and the coupling loss factor is computed from the numerical results.

\subsection{CLF calculation}

The CLF calculations are done for systems consisting of two subsystems. The SEA formulation in this case is

$$
\left\{\begin{array}{l}
\Pi_{1, \text { in }} \\
\Pi_{2, \text { in }}
\end{array}\right\}=\omega\left[\begin{array}{cc}
\eta_{11}+\eta_{12} & -\eta_{21} \\
-\eta_{12} & \eta_{21}+\eta_{22}
\end{array}\right]\left\{\begin{array}{l}
\left\langle E_{1}\right\rangle \\
\left\langle E_{2}\right\rangle
\end{array}\right\}
$$

where $\eta_{i i}$ and $\left\langle E_{i}\right\rangle$ are the internal loss factor and averaged energy of subsystem $i$ respectively, $\Pi_{i, \text { in }}$ is the input power in subsystem $i$ and $\eta_{i j}$ is the coupling loss factor between subsystems $i$ and $j$ (with $i \neq j$ ). This factor satisfies the consistency relationship

$$
\eta_{i j} n_{i}=\eta_{j i} n_{j}
$$

where $n_{i}$ is the modal density (number of modes per Hz) of subsystem $i$. 
One of the basic properties of the SEA formulation is the proportionality between the power exchanged by two subsystems $\Pi_{12}$ and the energies of these subsystems [17]

$$
\Pi_{12}=\omega\left(\eta_{12}\left\langle E_{1}\right\rangle-\eta_{21}\left\langle E_{2}\right\rangle\right)
$$

The standard procedure in SEA is to compute the averaged energies of the subsystems with Eq. (1). The input powers are usually known, for a given excitation. In building acoustics, the internal loss factor can be computed with analytical expressions available in the literature [4] for most types of subsystems. However, the analytical expression for the coupling loss factor is only available for simple connections.

In this work, the SEA formulation for a system consisting of two subsystems is used to estimate the coupling loss factor. The averaged energies are obtained from the numerical simulation of the same vibroacoustic problem and Eq. (1) is used to compute $\eta_{12}$. Since the energy values are frequency-dependent, the CLF will also depend on the frequency, and therefore the result of the computation will not be a single value but a CLF law in terms of the frequency.

Three different ways are explored for computing the coupling loss factor. Two of them are based on the SEA formulation for the 2-subsystem case (1), when only the first subsystem is excited (i.e. $\Pi_{2 \text {,in }}=0$ ). The third one is based on the power exchange at the connection (3). They are:

First expression: CLF is isolated from the first equation in system (1) (power balance of subsystem 1) as

$$
\eta_{12}=\frac{\Pi_{1, \text { in }} / \omega-\eta_{11}\left\langle E_{1}\right\rangle}{\left\langle E_{1}\right\rangle-\frac{n_{1}}{n_{2}}\left\langle E_{2}\right\rangle} .
$$

Second expression: CLF is isolated from the second equation in system (1) (power balance of subsystem 2) as

$$
\eta_{12}=\frac{\eta_{22}\left\langle E_{2}\right\rangle}{\left\langle E_{1}\right\rangle-\frac{n_{1}}{n_{2}}\left\langle E_{2}\right\rangle} .
$$

Third expression: CLF is isolated from the expression (3) of the power transmitted through the connection as

$$
\eta_{12}=\frac{\Pi_{12}}{\omega\left(\left\langle E_{1}\right\rangle-\frac{n_{1}}{n_{2}}\left\langle E_{2}\right\rangle\right)} .
$$

\section{$2.2 \quad$ Error propagation analysis}

The three techniques described in Section 2.1 involve operations between approximated quantities. Therefore, an analysis of the error propagation (see [13, 14]) is required in order to check the reliability of these calculations. The relative error of a certain approximation $\bar{x}$ with respect to the exact value $x$ can be computed as

$$
r_{x}=\frac{x-\bar{x}}{x}
$$

For the sake of simplicity, the following assumptions are made: 
- $\left\langle E_{1}\right\rangle \gg\left\langle E_{2}\right\rangle$. This is the weak coupling hypothesis, required by the SEA framework.

- $\eta_{11}=\eta_{22}$ and $n_{1}=n_{2}$. In all the examples both subsystems have the same properties, in particular the same internal loss factor and modal density. This assumption is not necessary but simplifies the expressions and facilitates the understanding of the error propagation.

- The internal loss factors $\eta_{i i}$ are given values and, therefore, they are errorfree. This hypothesis is removed in A, where a more complete error propagation analysis is performed, considering that every parameter may be subject to error.

If these simplifications are incorporated to Eq. (4), the value of the relative error of $\eta_{12}$ computed with the first expression is

$$
\begin{array}{r}
r_{\eta_{12}} \simeq\left(r_{\Pi_{1, \text { in }}}-r_{1}\right) \frac{\left(\Pi_{1, \text { in }}\right)}{\omega\left\langle E_{1}\right\rangle\left(\frac{\Pi_{1, \text { in }}}{\omega\left\langle E_{1}\right\rangle}-\eta_{11}\right)}=\left(r_{\Pi_{1, \text { in }}}-r_{1}\right)\left(1+\frac{\left\langle E_{1}\right\rangle \eta_{11}}{\frac{\Pi_{1, \text { in }}}{\omega}-\eta_{11}\left\langle E_{1}\right\rangle}\right)= \\
=\left(r_{\Pi_{1, \text { in }}}-r_{1}\right)\left(1+\frac{\left\langle E_{1}\right\rangle}{\left\langle E_{2}\right\rangle}\right)=\left(1+\frac{\left\langle E_{1}\right\rangle}{\left\langle E_{2}\right\rangle}\right) r_{\Pi_{1, \text { in }}}-\left(1+\frac{\left\langle E_{1}\right\rangle}{\left\langle E_{2}\right\rangle}\right) r_{1}
\end{array}
$$

where $r_{1}$ and $r_{\Pi_{1, \text { in }}}$ are the relative errors associated to the computation of $\left\langle E_{1}\right\rangle$ and $\Pi_{1, \text { in }}$ respectively.

The assumption of weak coupling leads to the conclusion that $\left|r_{\eta_{12}}\right| \gg\left|r_{\Pi_{1, \text { in }}}-r_{1}\right|$. The relative error of the CLF computed with this approach is larger than those of the energy and incoming power of subsystem 1 . There is an amplifying factor of the error caused by the subtraction of two very similar values, $\Pi_{1, \text { in }} / \omega$ and $\eta_{11}\left\langle E_{1}\right\rangle$, that makes this way of estimating the CLF unreliable.

The error committed in the computation of the CLF obtained with the second expression (5) is

$$
r_{\eta_{12}}=\frac{r_{2}-r_{1}}{1-r_{1}} \simeq r_{2}-r_{1}
$$

where $r_{2}$ is the relative error in the calculation of $\left\langle E_{2}\right\rangle$. The error in the CLF is of the same order of the errors in the calculation of the energies.

In a similar way, the value of the error committed with the third expression (6) is

$$
r_{\eta_{12}} \simeq r_{\Pi_{12}}-r_{1}
$$

where $r_{\Pi_{12}}$ is the relative error associated to the computation of $\Pi_{12}$. As in Eq. (9), the error in the CLF is of the order of the errors of the computed quantities, with no amplifying factor.

The relative error of the three expressions can be computed as a linear combination of the relative errors of the energies and powers. In Equations (9) and (10) the combination coefficients are equal to one, but in Eq. (8) they are much larger than one. The main conclusion is that subtractions of very similar quantities should be avoided in the computation of the CLF. Instead, expressions in which the power exchanged in the connection or the energy of the unexcited subsystem appear in the numerator provide better results. 
Two considerations must be done regarding these results. The first one is their dependence on the weak coupling between the subsystems. If the coupling is stronger, the first expression (4) performs much better in terms of the error propagation, because the two quantities in the subtraction are not similar anymore. However, the weak coupling is a hypothesis of SEA and, therefore, strong couplings lead to unreliable results with the three expressions.

The second consideration is related to the estimation of the CLF from experimental values. In that case, the experimental error may be a systematic error, and the values of $r_{\Pi_{1, \text { in }}}$ and $r_{1}$ may have the same sign and be of the same order. In that case, even if the coefficients that multiply the energy and power errors in Eq. (8) are much greater than one, the value of the two terms may be similar and therefore, they could compensate and provide a low value of the CLF error. This is one possible reason for the good results achieved in [3], where Campolina et al. estimate the coupling loss factor from experimental measurements. However, it is better to use the second expression, since it provides a more robust way for computing the CLF. In fact, this expression is equivalent to those resulting from the formulations in $[10,28]$ and the CLF expression derived in the Power Injection Method [16]. The third expression is also reliable, but it requires obtaining another value: the transmitted power.

\subsection{Deterministic approach}

The energies $\left\langle E_{1}\right\rangle$ and $\left\langle E_{2}\right\rangle$ and powers $\Pi_{1, \text { in }}$ and $\Pi_{12}$ required for the CLF calculation are obtained from numerical simulations of the vibroacoustic problems.

\subsubsection{Numerical modelling of the vibroacoustic problem}

Most of the examples consist of floors or walls connected by different devices. They are modelled with the thin plate equation in the frequency domain

$$
D \nabla^{4} u(x, y)-\omega^{2} \rho_{s} u(x, y)=q(x, y),
$$

expressing the vibration field in the plates in terms of the eigenfunctions of simply supported plates, see Díaz-Cereceda et al. [8]. In Eq. (11), $D=E h^{3} / 12\left(1-\nu^{2}\right)$ is the bending stiffness of the plate (where $E, \nu$ and $h$ are Young's modulus, Poisson's ratio and the thickness of the plate), $\rho_{s}$ its mass per unit surface, $q(x, y)$ the applied load per unit surface, $\omega=2 \pi f$ (with $f$ the frequency of vibration) and $u(x, y)$ the plate deflection.

If the plates are connected with a mechanical device, its effect is modelled as an extra force or moment, connecting the vibrations of both plates as explained in [8].

If the plates are connected by an air cavity forming a double wall, the coupled vibroacoustic problem is solved, namely the thin plate equation (11) for the plates and the Helmholtz equation

$$
\nabla^{2} p(\mathbf{x})+k^{2} p(\mathbf{x})=0
$$

for the acoustic domain, where $p(\mathbf{x})$ is the pressure field and $k$ is the wave number in the air. Modal analysis [9] is used for solving the acoustic part of the problem. This 
technique exploits the simple geometry of the problem to achieve an accurate result with less computational cost than a finite element discretisation. However, for a more complex geometry the problem might be solved with other discretisation techniques, such as the finite layer method [9] or the finite element method [23].

\subsubsection{Energy calculation}

The CLF estimation requires the computation of the averaged energy of each plate and, for the third expression, the power transmitted through the connection between plates.

Once the displacement field $u(x, y)$ in a plate is known, its velocity is obtained as $v(x, y)=\mathrm{i} \omega u(x, y)$, where $\mathrm{i}=\sqrt{-1}$. Then, the averaged energy of the plate is computed as [15]

$$
\langle E\rangle=M\left\langle v_{\mathrm{RMS}}^{2}\right\rangle
$$

where $M$ is the mass of the plate and $\left\langle v_{\mathrm{RMS}}^{2}\right\rangle$ is the spatial mean square value of its velocity.

According to SEA hypotheses, the incident field must be diffuse. For instance, if the excitation is an impact on one of the plates, the spatially distributed and uncorrelated excitation required by SEA is achieved by solving twenty different problems, each one with the force applied in a different (random) excitation point. The resulting energies are averaged to provide the diffuse output. The decision to use twenty different excitations was motivated by the works of Totaro and Guyader [31] and Maxit and Guyader [22]. Moreover, a convergence study has been performed, showing that the differences between using 20, 30 or 40 points are barely noticeable.

The computation of the power $\Pi_{12}$ transmitted between plates 1 and 2 through a connection depends on the connection characteristics. Its general expression is

$$
\Pi_{12}=\frac{1}{2} \operatorname{Re}\left(\int_{\Omega} q(x, y) v^{*}(x, y) \mathrm{d} \Omega\right)
$$

for a translational connection, or

$$
\Pi_{12}=\frac{1}{2} \operatorname{Re}\left(\int_{\Omega} m(x, y) w^{*}(x, y) \mathrm{d} \Omega\right)
$$

for a rotational connection; $m(x, y)$ is the moment per unit surface and $w(x, y)$ the rotational speed, both of them at the connection. The superscript $*$ means the conjugate of a complex number. This surface integral reduces to a line integral or a point evaluation for a line or point connection respectively.

The computation of the incoming power to the system is also necessary. If the excitation is a point force on one of the plates, the associated power is computed as

$$
\Pi_{\text {in }}=\frac{1}{2} \operatorname{Re}\left\{F_{p} v_{p}^{*}\right\}
$$

where the subscript $p$ means evaluated at the point where the impact is exerted and $F_{p}$ is the value of the applied force. 
However, if the excitation is a pressure wave impinging on one of the plates, the associated power is computed as

$$
\Pi_{\mathrm{in}}=\frac{\left\langle P_{\mathrm{RMS}}^{2}\right\rangle L_{x} L_{y} \cos \varphi}{\rho_{\mathrm{air}} c},
$$

where $\left\langle P_{\mathrm{RMS}}^{2}\right\rangle$ is the mean square pressure exciting the plate, $L_{x}$ and $L_{y}$ are the plate dimensions, $\varphi$ is the incidence angle of the pressure wave and $\rho_{\text {air }}$ and $c$ are the density and the speed of sound in the air respectively. This value is also averaged for different incident angles, ranging from $0^{\circ}$ to $90^{\circ}$ in order to reproduce the diffuse field excitation.

In some examples of this paper, the CLF between a room and an adjacent wall is also required. It has been computed numerically in order to capture as much information as possible. For the calculation, the energy of the room is computed assuming diffuse field as

$$
\langle E\rangle=V_{\text {room }}\left\langle P_{\mathrm{RMS}}^{2}\right\rangle / \rho_{\mathrm{air}} c^{2},
$$

where $V_{\text {room }}$ is the volume of the room.

\section{Applications in building acoustics}

\subsection{Coupling loss factor for mechanical connections}

In this section, some structural configurations with a known analytical expression of the CLF are studied. All the examples consist of walls or floors, which are modelled as thin plates, connected by mechanical devices.

The mechanical and geometric properties of the plates are summarised in Tables 1 and 2 respectively. The external excitation of the system is always a force of $1 \mathrm{~N}$, applied on plate 1 at every Hz. Therefore $\Pi_{j \text {,in }}=0$ for $j>1$.

The CLF calculations obtained for each $\mathrm{Hz}$ are averaged in third octave bands before plotting the result.

\begin{tabular}{lcl}
\hline Variable & Symbol & Value \\
\hline Young's modulus & $E$ & $2.5 \times 10^{9} \mathrm{~N} \mathrm{~m}^{-2}$ \\
Density & $\rho$ & $692.3 \mathrm{~kg} \mathrm{~m}^{-3}$ \\
Poisson's ratio & $\nu$ & 0.3 \\
Loss factor & $\eta$ & $3 \%$ \\
\hline
\end{tabular}

Table 1: Mechanical properties of the GN plasterboard plates.

\subsubsection{Double wall with connecting point springs}

The first example reproduces the case of a double wall without considering the effect of the air in the cavity. It consists of two parallel simply supported leaves connected with nine translational springs, equally distributed along the leaves as shown in Fig. 1. 


\begin{tabular}{lcl}
\hline Variable & Symbol & Value \\
\hline Plate size, $x$ direction & $L_{x}$ & $2.4 \mathrm{~m}$ \\
Plate size, $y$ direction & $L_{y}$ & $2.4 \mathrm{~m}$ \\
Thickness & $h$ & $0.013 \mathrm{~m}$ \\
\hline
\end{tabular}

Table 2: Geometric properties of the GN plasterboard plates.

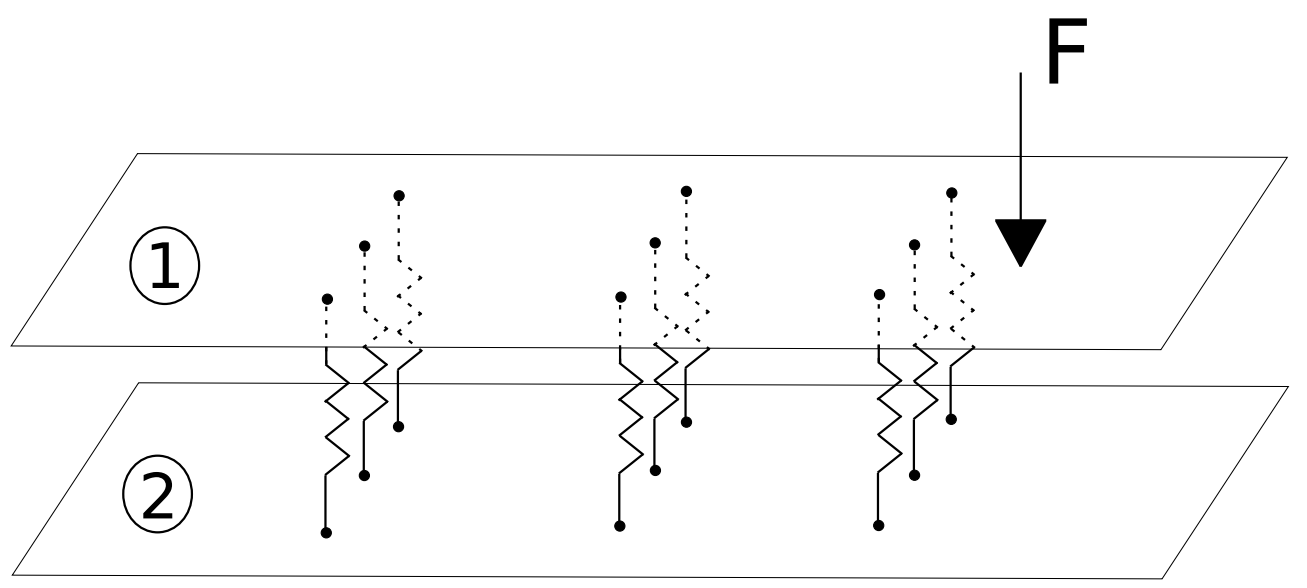

Figure 1: Distribution of the nine springs.

These springs represent the effect of studs connected by point screws to the leaves. The stiffness of each spring is $K=8 \cdot 10^{5} \mathrm{~N} \mathrm{~m}^{-1}$.

The CLF estimations have been compared with an analytical expression typically used in SEA [15, 4], obtained by analogy with an electrical circuit and valid when each connection acts independently of the others

$$
\eta_{12}=\frac{n \operatorname{Re}\left\{Y_{2}\right\}}{\omega M_{1}\left|Y_{1}+Y_{2}+Y_{s}\right|^{2}}
$$

In Eq. (19),

$$
Y_{i}=\frac{1}{8 \sqrt{D_{i} \rho_{s i}}} \quad i=1,2
$$

is the point mobility of each leaf, $M_{1}$ is the mass of leaf 1 ,

$$
Y_{s}=\frac{i \omega}{K}
$$

is the mobility of the spring and $n=9$ is the number of springs used.

In Fig. 2 the three methods described in Section 2.1 for computing the CLF are compared with the analytical expression.

For this simple problem, the numerical estimations of the CLF provide good results for the second and third expressions. However, results derived from the first expression (4) are wrong. This behaviour is explained with the analysis of error propagation shown in Section 2.2, and confirms the unreliability of this expression for obtaining the CLF, specially when the coupling between the leaves is very weak. This is illustrated 


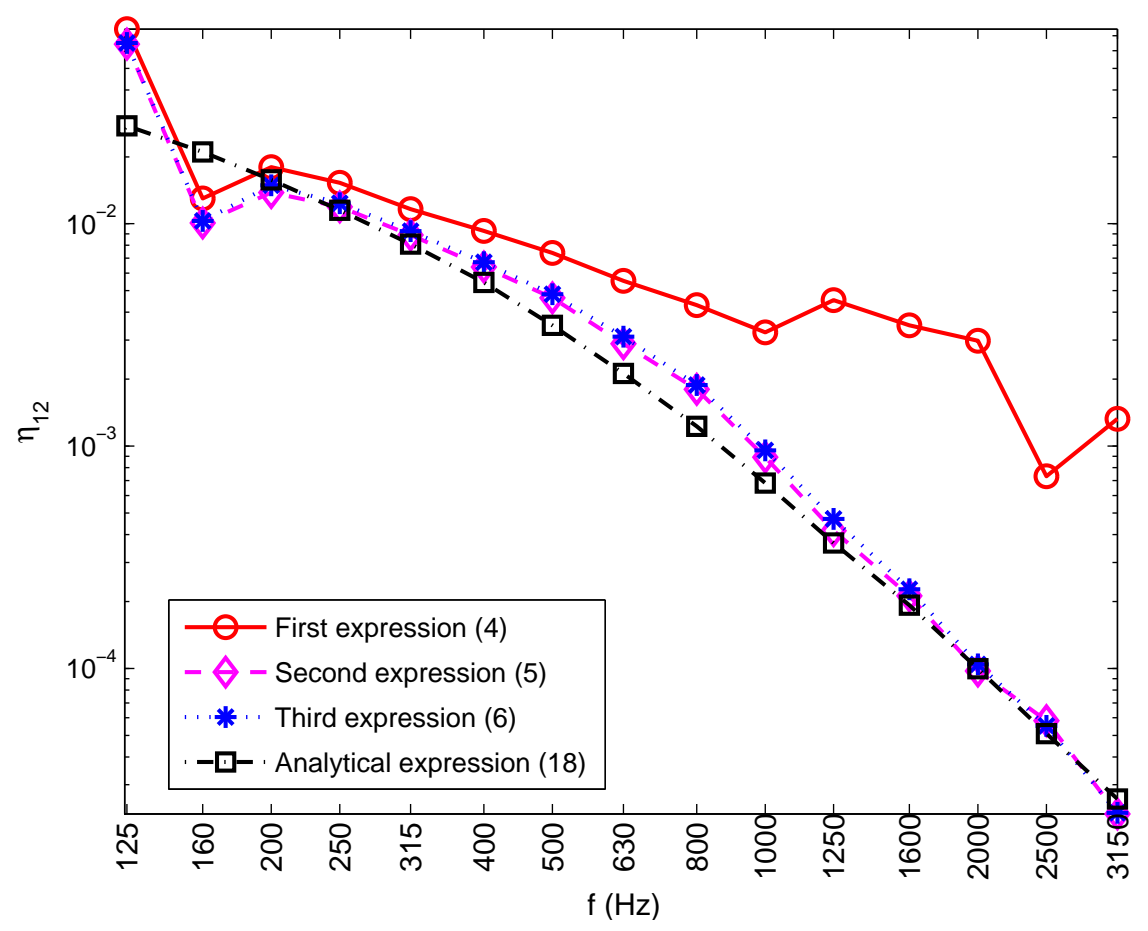

Figure 2: Comparison of the three methods of estimating $\eta_{12}$ and its analytical expression for nine spring connections.

in Fig. 3, where the evolution of the ratio $\left\langle E_{1}\right\rangle /\left\langle E_{2}\right\rangle$ with the frequency is shown. The figure shows that the larger the frequency, the larger the amplifying factor. This is coherent with the weaker behaviour of the coupling when the frequency increases, and explains the large error of the first expression, specially at the highest frequencies.

The differences at low frequencies are reasonable, since most of the SEA hypotheses are not satisfied at those frequencies and therefore the SEA-based expressions are no longer valid.

\subsubsection{Rotational joint}

The second example consists of two adjacent floors with a rotational joint in the common edge, see Fig. 4. It reproduces the effect of elastic joints through a long floor. The rotation stiffness of the joint is $K_{\theta}=10^{3} \mathrm{~N} \mathrm{~m} / \mathrm{rad} \mathrm{m}$. It relates the plate rotations $\theta$ and bending moments $m$ on both sides of the joint as $m_{1}=m_{2}=K_{\theta}\left(\theta_{1}-\theta_{2}\right)$, [8].

The CLF estimated from the numerical solution has been compared with an analytical expression obtained with the wave approach [7]. It is based on the orthogonality between the joint and the waves propagating in the floor. The vibration field in the excited floor span (subsystem 1) is assumed to consist of incident waves, reflected waves in the far field (propagating) and reflected waves in the near field (evanescent). The vibration field in the span with no external excitation consists of transmitted waves, both in the near and the far field. The expressions of the vibration fields in 


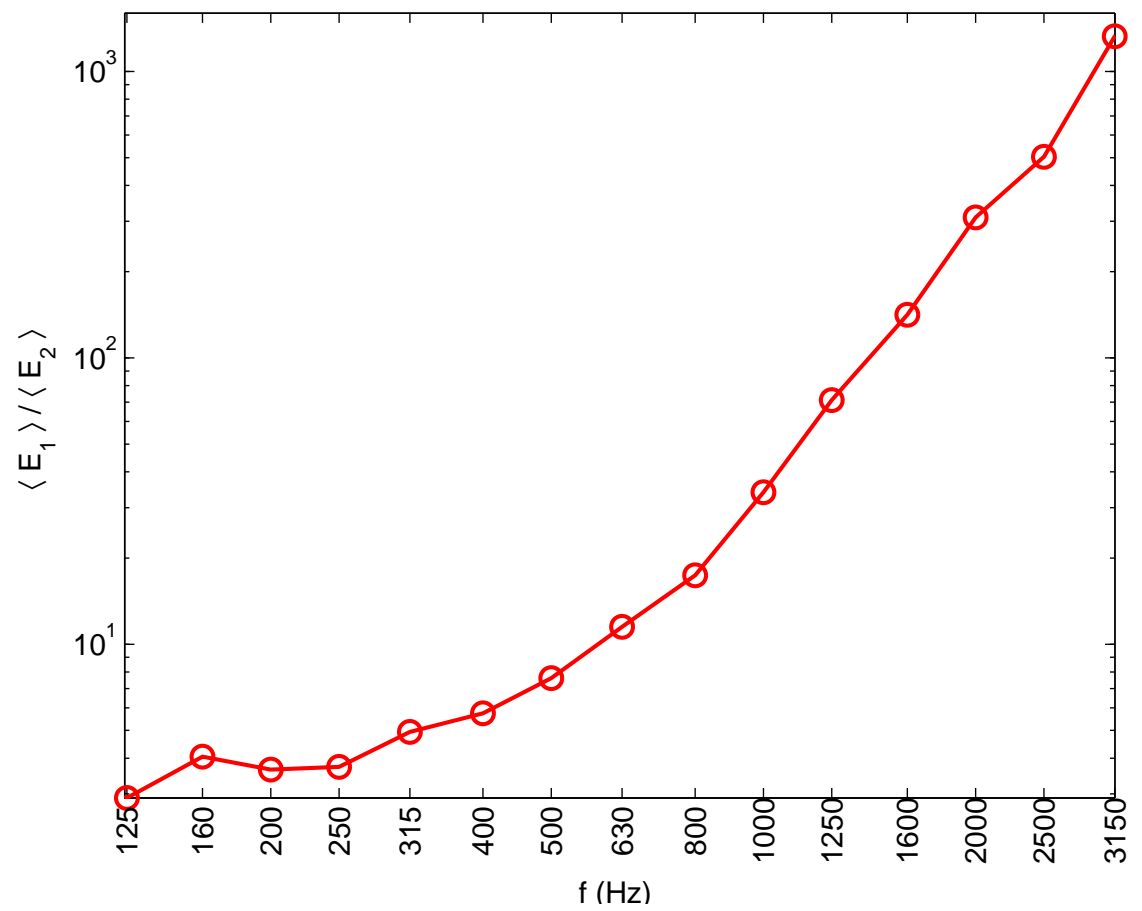

Figure 3: Evolution of the amplification factor of the error with the frequency.



Figure 4: Sketch of the rotational joint.

spans 1 and $2\left(v_{z 1}\right.$ and $\left.v_{z 2}\right)$ are

$$
\begin{aligned}
& v_{z 1}=v_{1}\left(\mathrm{e}^{-\mathrm{i} k_{1} y}+r \mathrm{e}^{\mathrm{i} k_{1} y}+r_{i} \mathrm{e}^{k_{1} y}\right) \\
& v_{z 2}=v_{1}\left(t \mathrm{e}^{-\mathrm{i} k_{2} y}+t_{i} \mathrm{e}^{-k_{2} y}\right)
\end{aligned}
$$

where $k_{1}$ and $k_{2}$ are the wave numbers in spans 1 and 2 respectively, $r$ and $t$ are the reflection and transmission parameters and $r_{i}$ and $t_{i}$ their equivalents in the near field.

In order to find $r, t, r_{i}$ and $t_{i}$ the boundary conditions at the contact line of both spans are applied

$$
v_{z 1}=0 ; \quad v_{z 2}=0 ; \quad m_{1}=m_{2}=K_{\theta}\left(\theta_{1}-\theta_{2}\right) .
$$

Assuming that the wave numbers and bending stiffnesses are the same for both 
spans $k_{1}=k_{2}=k$ and $B_{1}=B_{2}=B$, the following linear system holds:

$$
\left[\begin{array}{cccc}
1 & 1 & 0 & 0 \\
0 & 0 & 1 & 1 \\
-1 & 1 & 1 & -1 \\
\mathrm{i} & 1 & \mathrm{i}-\frac{B k}{K_{\theta}} & 1+\frac{B k}{K_{\theta}}
\end{array}\right]\left\{\begin{array}{c}
r \\
r_{i} \\
t \\
t_{i}
\end{array}\right\}=\left\{\begin{array}{c}
-1 \\
0 \\
1 \\
\mathrm{i}
\end{array}\right\} .
$$

From this system, the value of $r$ is obtained and therefore the transmission coefficient can be computed as $\tau_{12}=1-|r|^{2}$. The CLF is obtained with the expression for two plates sharing one edge

$$
\eta_{12}=\frac{2 c_{B} L \tau_{12}}{\pi \omega S}
$$

where $c_{B}=\sqrt{\omega^{2} D / \rho_{s}}$ is the propagation velocity of the waves in the plates, $L$ is the length of the common edge and $S$ is the surface of one plate.

In Fig. 5 the comparison between the three estimation methods described in Section 2.1 and this analytical approximation is shown.

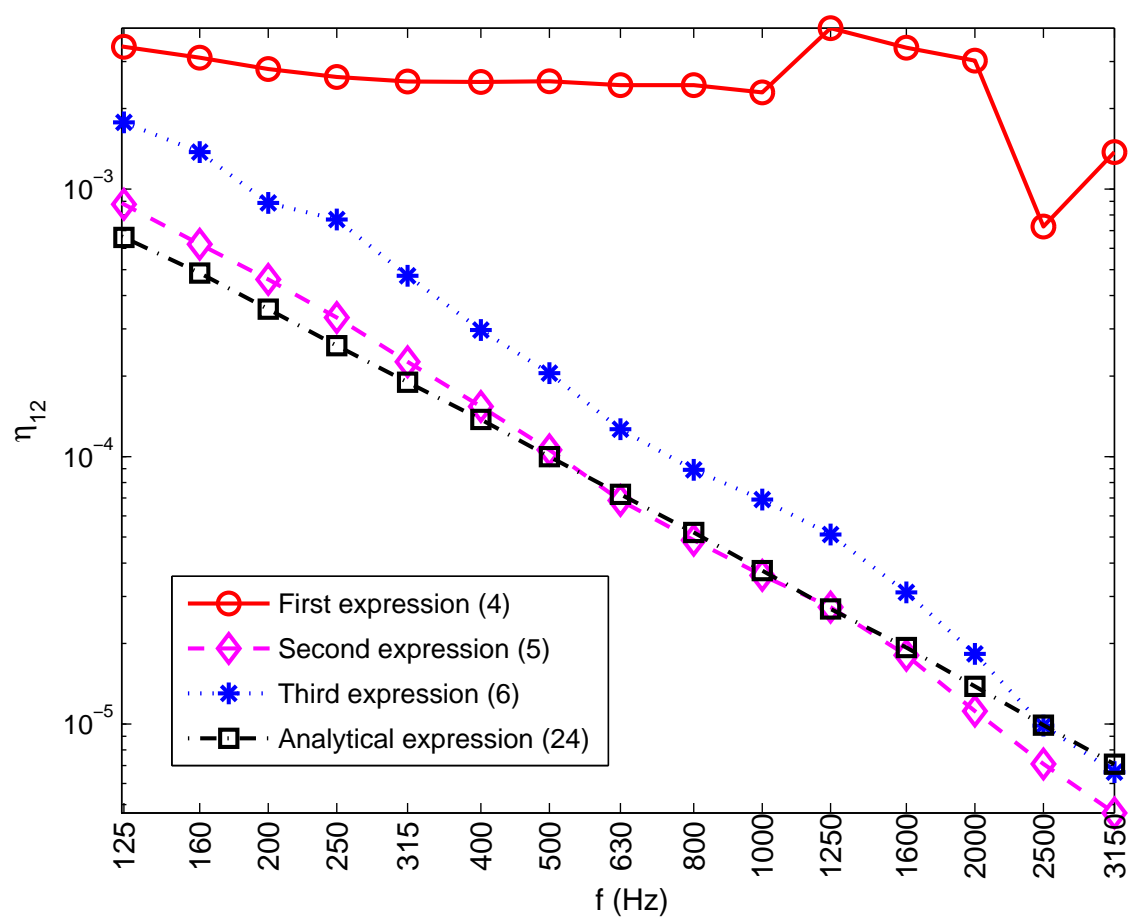

Figure 5: Comparison of the $\eta_{12}$ estimations and its analytical expression for the rotational joint.

Again the errors with the first expression (4) are unacceptable, while the other techniques present the same trend as the analytical one. However, there are some differences between the results obtained with the second and third expressions. A possible explanation for this discrepancy is that the computation of the power transmitted at the joint is less trustable than that of the example of Section 3.1.1. In this case, the power must be computed along a line and is also affected by the boundary 
conditions of the plate (the joint is located at an edge of the span). Therefore, the estimation of the CLF from the power at the connection (third expression) is less accurate than in the previous example.

\subsubsection{Application for long floors}

With the CLF estimations for the rotational joint, a new configuration is simulated: the propagation of vibrations through a long floor consisting of four simply supported plates linked together with elastic joints as shown in Fig. 6.

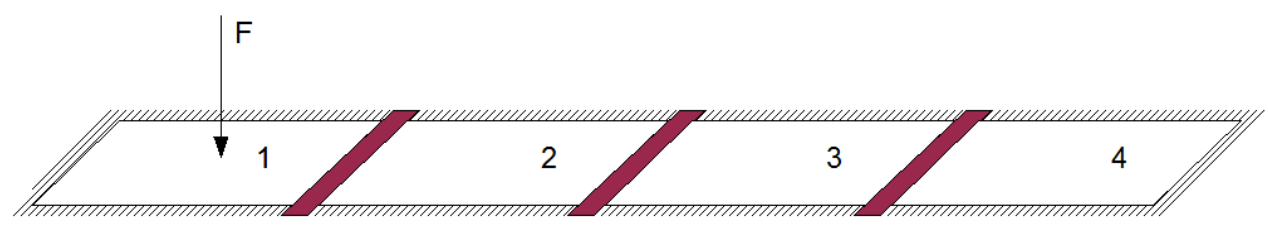

Figure 6: Sketch of the four plates linked with rotational joints.

The goal is to check whether the CLF obtained in Section 3.1.2 for the rotational joint can be used in the SEA simulation of a structure consisting of more than two subsystems, i.e. more than two floor spans. This is an example of the potential of the technique proposed in this paper: obtaining the coupling loss factor between two subsystems with a deterministic computation and applying it for solving larger problems with SEA.

In order to perform the full statistical energy analysis, the only information needed, in addition to the coupling loss factor, are the internal loss factors of the subsystems and the input power. The internal loss factor is the same for all the subsystems and is defined in Table $1\left(\eta_{i i}=\eta\right)$. The input power is computed as $\Pi_{1, \text { in }}=\frac{1}{2}\left|F_{1}\right|^{2} \operatorname{Re}\left\{1 / Y_{1}\right\}$ because the velocity field is not known a priori.

Using the CLF $\eta_{12}^{\text {num }}$ obtained in Section 3.1.2 for every joint and assuming that all the connections and subsystems are equal $\left(\eta_{i j}=\eta_{12}^{\text {num }} \forall i, j\right)$, the energies of every subsystem are obtained from the global SEA system

$$
\left\{\begin{array}{c}
\Pi_{1, \text { in }} \\
0 \\
0 \\
0
\end{array}\right\}=\omega\left[\begin{array}{cccc}
\eta+\eta_{12}^{\text {num }} & -\eta_{12}^{\text {num }} & 0 & 0 \\
-\eta_{12}^{\text {num }} & \eta+2 \eta_{12}^{\text {num }} & -\eta_{12}^{\text {num }} & 0 \\
0 & -\eta_{12}^{\text {num }} & \eta+2 \eta_{12}^{\text {num }} & -\eta_{12}^{\text {num }} \\
0 & 0 & -\eta_{12}^{\text {num }} & \eta+\eta_{12}^{\text {num }}
\end{array}\right]\left\{\begin{array}{c}
\left\langle E_{1}\right\rangle \\
\left\langle E_{2}\right\rangle \\
\left\langle E_{3}\right\rangle \\
\left\langle E_{4}\right\rangle
\end{array}\right\} .
$$

The vibration level difference between the first and the fourth floor spans

$$
D_{14}=10 \log \frac{\left\langle E_{1}\right\rangle}{\left\langle E_{4}\right\rangle}
$$

obtained with SEA is compared in Fig. 7 with the numerical solution obtained for the deterministic analysis of the four plates. The results obtained with SEA are computed using the two best CLF laws estimated in the 2-subsystem case: the second and third expressions (Eqs. (5) and (6) respectively). The trend of $D_{14}$ is well captured with 
SEA, for both laws of the CLF. For the SEA computation, a tridiagonal $4 \times 4$ problem is solved at each frequency, while the linear systems to be solved in the numerical computation are block-tridiagonal and their size ranges from 624 degrees-of-freedom (dof) for the lowest frequency to 968 dof for the highest frequency.

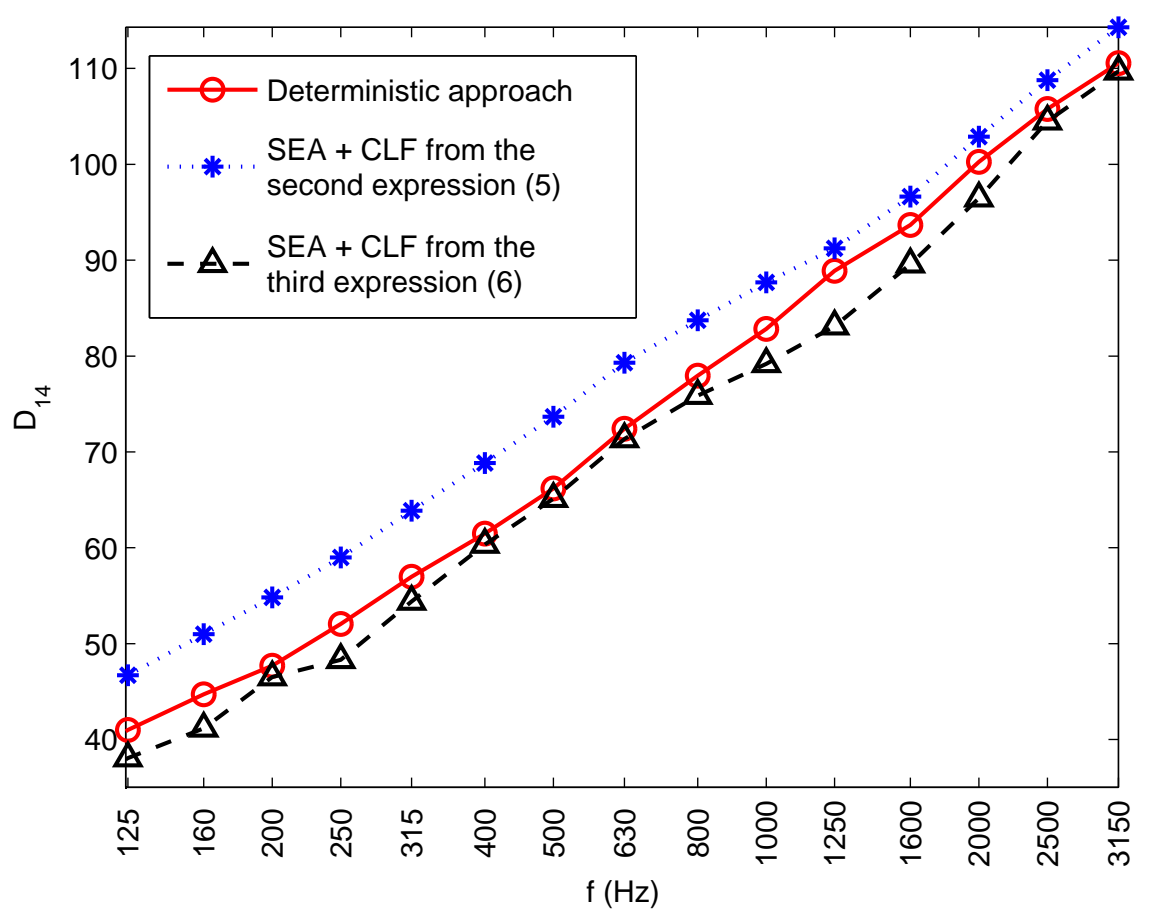

Figure 7: Comparison of $D_{14}$ obtained with the deterministic and the statistical methods using the CLF obtained numerically.

\subsection{Coupling loss factor in double walls}

The isolating effect of the air cavity located between the two leaves of a double wall is analysed here. This example has been chosen because it is a representative vibroacoustic problem and also because the SEA treatment of double walls is a challenge: the air cavity may be treated as an SEA subsystem or just as a connection between subsystems [15].

The use of SEA for modelling double walls has been studied by Craik in [5] and [6] and by Brekke in [2]. Craik focuses on the search of accurate expressions for the indirect coupling loss factor between the rooms and the cavity. On the other hand, Brekke concludes, with the help of experiments, that the effect of the air stiffness should be taken into account, as well as the resonant transmission, for lower frequencies.

Here, the coupling loss factor between the two leaves of the double wall is computed numerically and compared with analytical expressions available in the literature. Then, an example of the sound reduction index between two rooms separated by a double wall is reproduced with statistical energy analysis using the coupling loss factors estimated from numerical simulations. This result is compared with the sound 
reduction index computed with a purely deterministic approach. Finally, the effect of steel studs in the sound reduction index of the double wall is analysed. The same example is reproduced adding studs of different shapes, and their coupling effect is compared with that of the air cavity.

For all the examples, the leaves are made of plasterboard, whose properties are described in Table 1 and the cavity thickness is $H=0.07 \mathrm{~m}$. Therefore, the massair-mass natural frequency for the two leaves and the cavity is $f_{\text {mam }}=106.5 \mathrm{~Hz}$. Moreover, the absorption coefficient at the cavity contour is assumed to be $\alpha=0 \%$. This is a reasonable assumption, since the area of the cavity contour is much lower than the rest of areas involved in the problem, and therefore the dissipation at the cavity is negligible compared to the energy transmissions. If the cavity was filled with absorbing material, the behaviour would be much different and the cavity should be treated as a non-conservative connection between the two leaves. This topic will be addressed in a forthcoming contribution.

\subsubsection{Comparison with analytical expressions}

Different SEA references [4, 15] do not coincide in the optimal way to deal with double walls in SEA. Neither the identification of subsystems nor the coupling loss factor expressions are clear.

A very common option is to consider the cavity as an SEA subsystem [4] (see Fig. 8), and obtain its own modal density

$$
n_{i}=\frac{4 \pi f^{2} V_{\mathrm{cav}}}{c^{3}}+\frac{2 \pi f S_{\mathrm{cav}}}{4 c^{2}}+\frac{L_{\mathrm{cav}}}{8 c}
$$

and internal loss factor

$$
\eta_{i i}=\frac{c \alpha S_{\mathrm{cav}}}{8 \pi f V_{\mathrm{cav}}}
$$

where $V_{\text {cav }}$ is the cavity volume, $S_{\text {cav }}$ is the surface of the cavity boundary (the sum of the area of all the faces surrounding the cavity except the contact surface with the leaves) whose absorbing factor is $\alpha$ and $L_{\text {cav }}$ is the sum of the lengths of the twelve cavity edges. In the examples of this work, $\eta_{i i}=0$.

Then, the coupling loss factors between the cavity and the leaves are obtained as

$$
\eta_{i j}=\frac{\rho_{\mathrm{air}} c \sigma f_{c}}{4 \pi f^{2} \rho_{s}}
$$

where $f_{c}$ is the coincidence frequency between the leaf and the air and $\sigma$ is the radiation efficiency of the leaf. The radiation efficiency is computed with the expressions defined in [20] with a small modification: the critical frequency treatment is applied for all the frequencies in a range of $f_{c} \pm 5 \mathrm{~Hz}$.

For this case, an equivalent coupling loss factor between the leaves is obtained in order to compare it with the other techniques. This is possible because the absorption coefficient at the cavity is null and, therefore, the cavity only transmits energy, without dissipating it. 


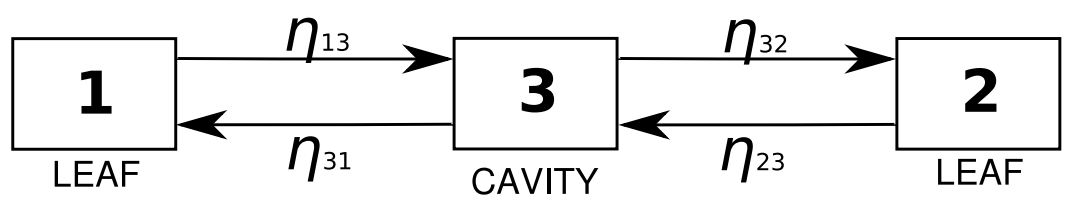

Figure 8: Sketch of an SEA model of a double wall where the cavity is considered as a subsystem.

Considering the cavity as subsystem 3, the global SEA-system is

$$
\left\{\begin{array}{c}
\Pi_{1, \text { in }} \\
0 \\
0
\end{array}\right\}=\omega\left[\begin{array}{ccc}
\eta_{11}+\eta_{13} & -\eta_{31} & 0 \\
-\eta_{13} & \eta_{33}+\eta_{31}+\eta_{32} & -\eta_{23} \\
0 & -\eta_{32} & \eta_{22}+\eta_{23}
\end{array}\right]\left\{\begin{array}{l}
\left\langle E_{1}\right\rangle \\
\left\langle E_{3}\right\rangle \\
\left\langle E_{2}\right\rangle
\end{array}\right\}
$$

Assuming that the two leaves are identical $\left(\eta_{13}=\eta_{23}, \eta_{31}=\eta_{32}\right.$ and $\left.\eta_{11}=\eta_{22}\right)$ and imposing $\eta_{33}=0$, the system becomes

$$
\left\{\begin{array}{c}
\Pi_{1, \text { in }} \\
0 \\
0
\end{array}\right\}=\omega\left[\begin{array}{ccc}
\eta_{11}+\eta_{13} & -\eta_{31} & 0 \\
-\eta_{13} & 2 \eta_{31} & -\eta_{13} \\
0 & -\eta_{31} & \eta_{11}+\eta_{13}
\end{array}\right]\left\{\begin{array}{c}
\left\langle E_{1}\right\rangle \\
\left\langle E_{3}\right\rangle \\
\left\langle E_{2}\right\rangle
\end{array}\right\} .
$$

Isolating $\left\langle E_{3}\right\rangle$ from the second equation and replacing it in the other two, the system can be reduced to

$$
\left\{\begin{array}{c}
\Pi_{1, \text { in }} \\
0
\end{array}\right\}=\omega\left[\begin{array}{cc}
\eta_{11}+\eta_{13} / 2 & -\eta_{13} / 2 \\
-\eta_{13} / 2 & \eta_{11}+\eta_{13} / 2
\end{array}\right]\left\{\begin{array}{c}
\left\langle E_{1}\right\rangle \\
\left\langle E_{2}\right\rangle
\end{array}\right\}
$$

and, therefore, the equivalent value of $\eta_{12}$ is

$$
\eta_{12}^{\text {equi }}=\frac{\eta_{13}}{2}
$$

Another option is to consider the air cavity as a connection between the two subsystems (leaves) as shown in Fig. 9; in particular as a spring with stiffness $K_{\text {air }}=$ $\rho_{\text {air }} c^{2} S / H$, where $H$ is the thickness of the cavity. This approach takes into account the non-resonant transmission caused by the stiffness of the air. The coupling loss factor is computed with the electrical circuit analogy, as done by Hopkins [15], using Eq. (19) with $n=1$.

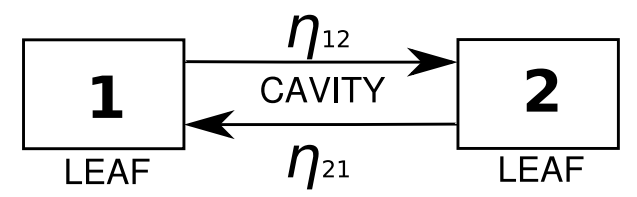

Figure 9: Sketch of an SEA model of a double wall where the cavity is considered as a connection.

In this work, the coupling loss factor between the two leaves of a double wall is obtained solving the vibroacoustic problem with modal analysis and isolating the CLF 


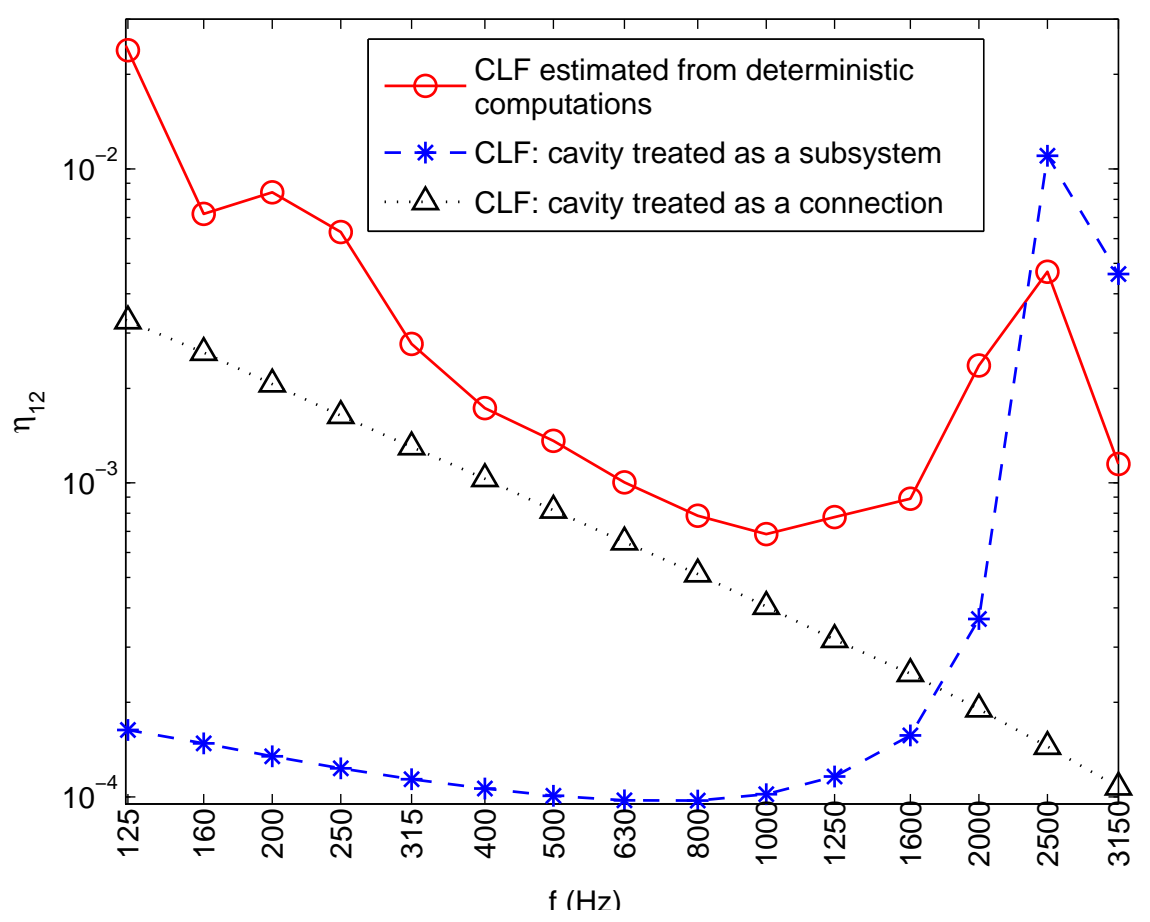

Figure 10: Comparison of the $\eta_{12}$ estimations and analytical expressions for the cavity in double walls.

with the second expression (5). This CLF law is compared with the two analytical approaches in Fig. 10. The dimensions of the leaves are the same as in Table 2.

Leaving the low-frequency discrepancies aside, Fig. 10 shows that the numerical estimation of the CLF is a good way of taking into account all the physical phenomena occurring in the wall. It shows, as Brekke suggested in [2], two main features: on the one hand, the importance of the equivalent stiffness of the air, specially at mid frequencies; on the other hand, the coincidence phenomenon that takes place at 2500 $\mathrm{Hz}$ in this double wall. This phenomenon is only considered by SEA when the cavity is treated as a subsystem. In fact, SEA overestimates a little the transmission at that frequency. This may be due to the simplifications involved in the expressions of the radiation efficiency. Therefore, both analytical expressions miss part of the physical information. However, if the analytical expressions are to be used, a complete SEA model is recommended, considering both transmission paths in order to reproduce the real behaviour. The cavity must be considered both as a connecting device and as a subsystem (see Fig. 11).

In Fig. 12 the coupling loss factor obtained numerically is compared with the CLF resulting from adding the coupling loss factor between the two leaves computed with the electrical analogy and the equivalent coupling loss factor between the leaves described in Eq. (34). The need of considering both behaviours together along the whole frequency range becomes evident. 


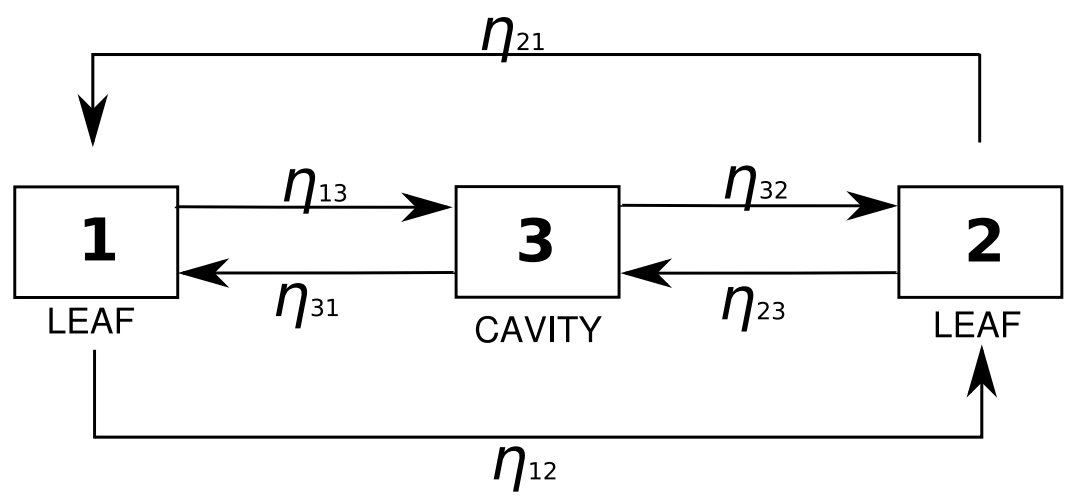

Figure 11: SEA sketch for the combination of the two techniques.

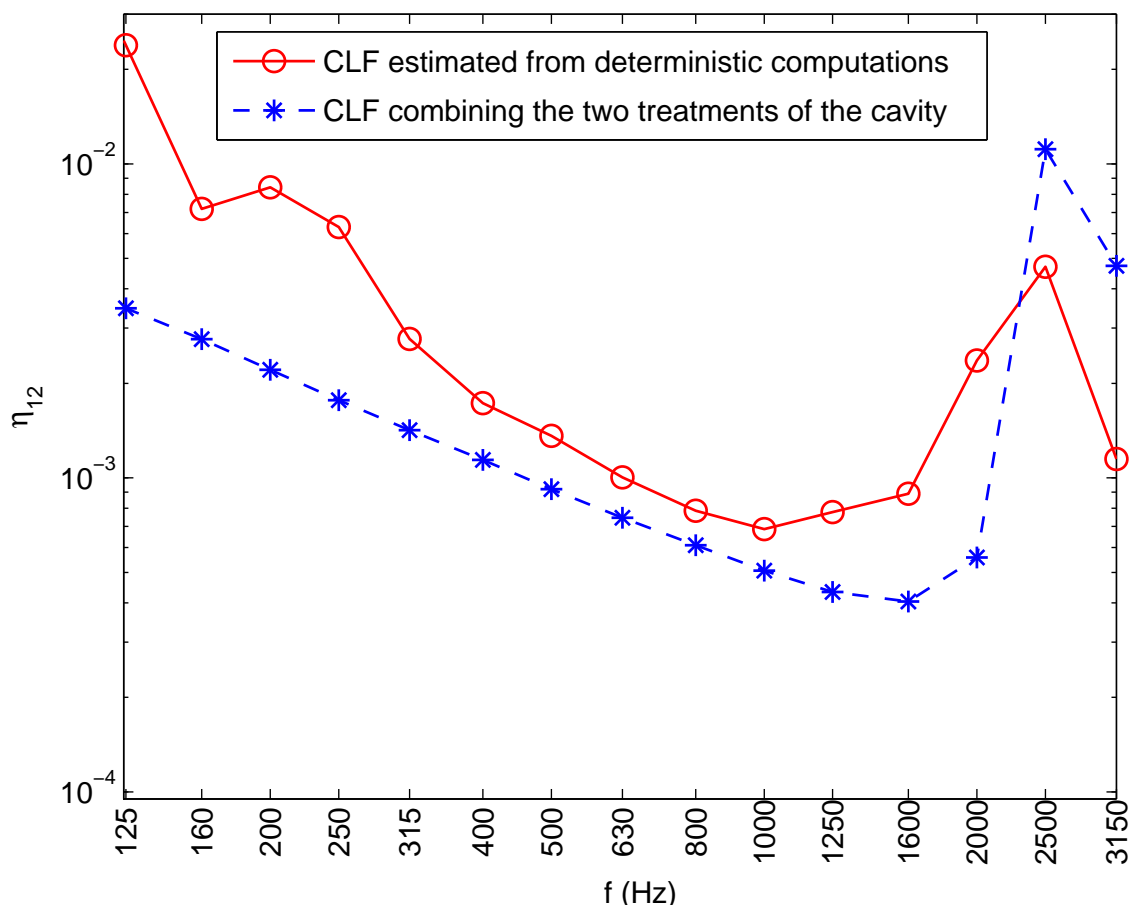

Figure 12: Comparison of the $\eta_{12}$ estimations and the combination of analytical expressions for the cavity in double walls.

\subsubsection{Application for larger vibroacoustic problems}

The potential of this technique for dealing with large vibroacoustic problems is shown computing with SEA the sound reduction index between two rooms separated by a double wall (Fig. 13). The coupling loss factors required for the analysis are estimated from numerical simulations. The sound reduction index is compared with the one obtained from the deterministic approach to the same problem. In this simulation, the properties of the system correspond to those of a proper-SEA system [19]. If, on the contrary, the system is quasi-SEA and indirect coupling loss factors are required, 
they can be estimated as described in B.

The sound reduction index is obtained from the mean square pressures at the source $\left(P_{\mathrm{RMS}_{1}}^{2}\right)$ and receiving $\left(P_{\mathrm{RMS}_{2}}^{2}\right)$ rooms as

$$
R=10 \log _{10} \frac{P_{\mathrm{RMS}_{1}}^{2}}{P_{\mathrm{RMS}_{2}}^{2}}+10 \log _{10} \frac{S}{A} .
$$

where $S$ is the surface of the wall and $A=\sum \alpha_{j} S_{j}$ is the absorption area in the receiving room.

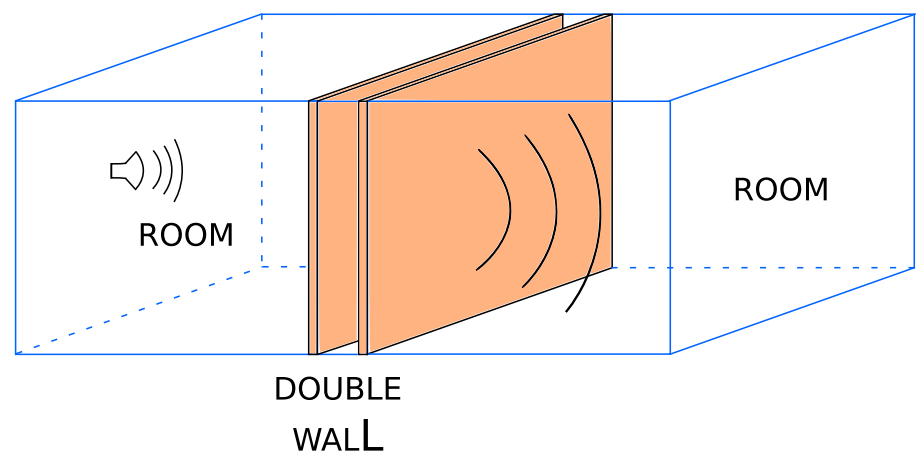

Figure 13: Sketch of the sound transmission through a double wall.

The deterministic approach is based on solving numerically the Helmholtz equation (12) for the rooms and using the analysis described in Section 2.3.1 for the double wall.

The SEA approach consists of 4 subsystems: sending room, leaf 1, leaf 2 and receiving room. The coupling loss factors between the two leaves $\eta_{23}$ and $\eta_{32}$ are obtained from the results of the deterministic computation described in Section 3.2.1. The coupling loss factors between each leaf and the adjacent room have been computed with numerical simulations too, because the expressions provided in [20] for the radiation efficiency are only suitable for mechanical excitations. Therefore, they underestimate the sound transmission when the excitation consists of a diffuse pressure field, as shown by Vigran on [32].

The leaf-room CLF is obtained with Eq. (5), computing the averaged energies from the numerical simulation of a system consisting of a room in contact with a leaf (see Fig. 14). Since the first SEA subsystem is the sending room and the last one is the receiving room, the influence of applying the correct excitation when estimating the coupling loss factor between the room and the leaf is studied.

Two vibroacoustic problems have been solved, with the only difference of the excitation applied to the system. In problem $\mathrm{A}$, the excitation is a sound source in the room (left part of Fig. 14). In problem $\mathrm{B}$, the excitation is a pressure wave impinging on the leaf (right part of Fig. 14). A natural approach would be to estimate $\eta_{12}$ and $\eta_{21}$ from problem $\mathrm{A}$ and $\eta_{34}$ and $\eta_{43}$ from problem B. However, the influence of this choice is analysed here.

In Fig. 15 the sound reduction index between the two rooms is shown from three different approaches. Two of them correspond to solving the problem with SEA. On 

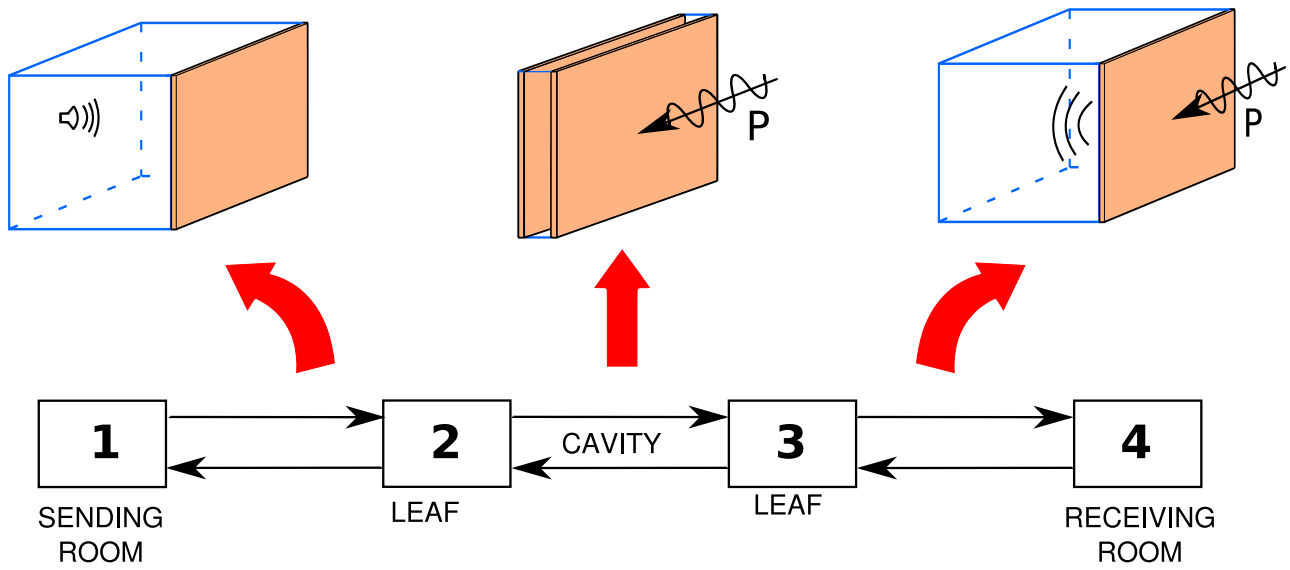

Figure 14: Sketch of the 2-subsystem vibroacoustic problems solved to obtain the coupling loss factors.

the first one, $\eta_{12}$ and $\eta_{21}$ are estimated from problem A, and $\eta_{34}$ and $\eta_{43}$ from problem B. On the second one, the four coupling loss factors between the rooms and leaves are obtained from problem $\mathrm{B}$. The third one is the result of a numerical calculation of the sound reduction index with a full deterministic approach [11], based on modelling the rooms with Helmholtz equation and the double wall as described in Section 2.3.1.

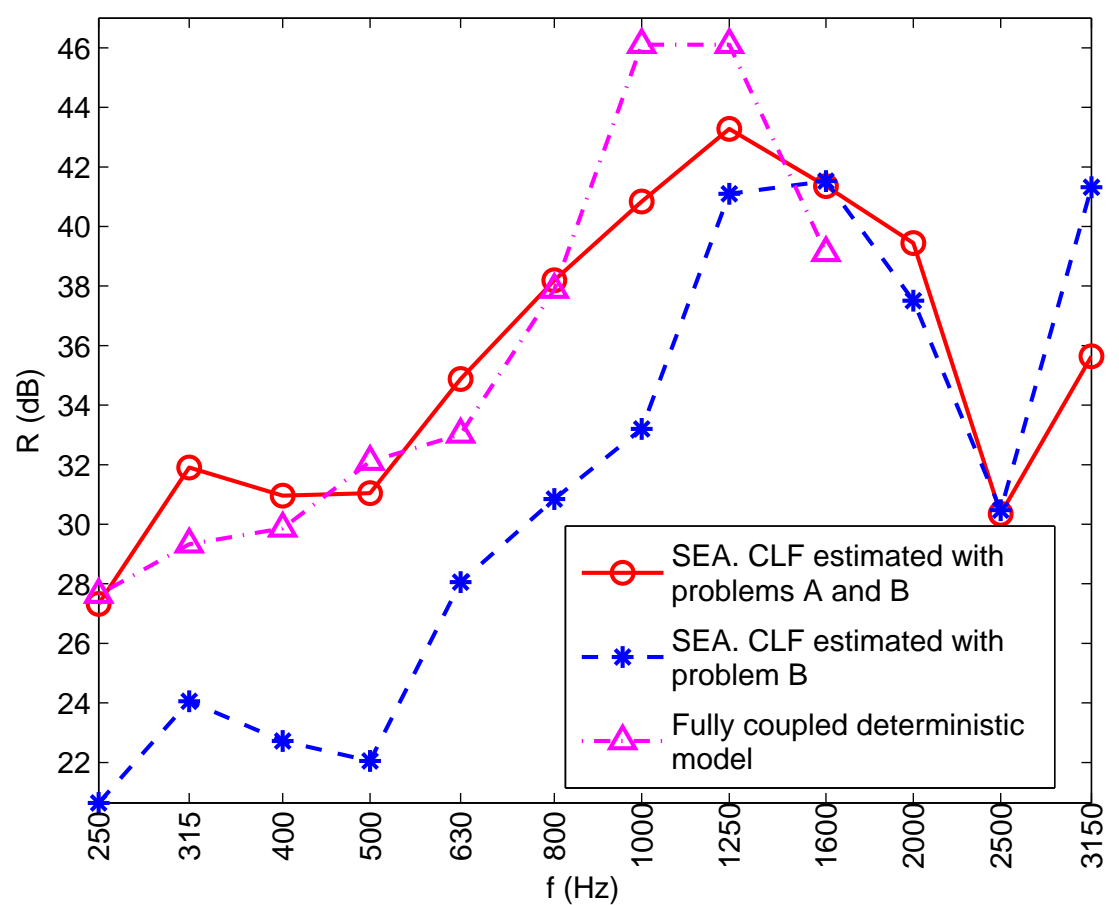

Figure 15: Comparison of the sound reduction index between two rooms separated by a double wall. 
In this example, the two rooms are identical, as well as the two GN plasterboard leaves. Their material properties are enumerated in Table 1 and the rest of the problem data are described in Table 3.

\begin{tabular}{lll}
\hline Variable & Symbol & Value \\
\hline Plate size, $x$ direction & $L_{x}$ & $2 \mathrm{~m}$ \\
Plate size, $y$ direction & $L_{y}$ & $3 \mathrm{~m}$ \\
Plate thickness & $h$ & $0.013 \mathrm{~m}$ \\
Cavity thickness & $H$ & $0.07 \mathrm{~m}$ \\
Room dimensions & $L_{x} \times L_{y} \times L_{z}$ & $2 \mathrm{~m} \times 3 \mathrm{~m} \times 5 \mathrm{~m}$ \\
Room absorption & $\alpha$ & $10 \%$ \\
Cavity absorption & $\alpha_{\text {cav }}$ & $0 \%$ \\
\hline
\end{tabular}

Table 3: Sound reduction index through the double plasterboard wall. Problem data.

Results in Fig. 15 are not shown for the lowest frequencies because SEA hypotheses are not satisfied there. The sound reduction index law computed numerically, or with SEA and the CLFs obtained from problems A and B, present the same trend. However, the trend of the results computed only with the CLFs estimated from problem B is much different than the other two. This illustrates the importance of estimating the coupling loss factors from problems with the same excitation at which the subsystems will be subjected afterwards.

The computation of the sound reduction index with the deterministic approach could not be performed for the highest frequencies, due to the large number of degrees of freedom (more than 65 000) involved in the numerical computation at each frequency. The improvement of the proposed approach in terms of the computational cost is remarkable, since the SEA approach only requires solving a $4 \times 4$ linear system at each frequency, and the numerical simulations required for estimating the coupling loss factors are much smaller than the full coupled approach. Furthermore, once the coupling loss factors are computed, they can be used in the SEA resolution of any other problem consisting of repetitions of the same elements (rooms and walls), such as a whole building.

\subsubsection{Effect of the studs in the sound transmission through double walls}

The acoustic effect of steel studs located between the two leaves of the double wall is studied with SEA, Fig. 16. To do so, the coupling loss factor associated to the stud connections is computed and added to that of the air cavity to reproduce the global behaviour of the double wall.

The computation of the coupling loss factor associated to the studs is performed assuming them to be line springs with frequency-dependent stiffnesses. These stiffness laws were computed from numerical simulations by Poblet-Puig et al. in [24]. If the connections were more complex and could not be approximated by line springs, a finite element analysis would have to be performed, see Fig. 17, and the CLF would be computed with one of the expressions described in Section 2.1. 
Given the equivalent stiffness of the stud, the associated coupling loss factor can be derived similarly as in [24]

$$
\eta_{12}=\frac{n_{L} \operatorname{Re}\left\{Y_{2}^{L} / L_{y}\right\}}{\omega M_{1}\left|Y_{1}^{L} / L_{y}+Y_{2}^{L} / L_{y}+Y_{s}^{L} / L_{y}\right|^{2}},
$$

where $n_{L}$ is the number of studs; the line mobilities of the leaves, modelled as thin plates, are obtained as [27]

$$
Y^{L}=\frac{1}{2(1+\mathrm{i}) \rho_{s} c_{B}\left(f / f_{c}\right)^{1 / 2}},
$$

and the line mobility of the spring is $Y_{s}^{L}=\mathrm{i} \omega / K_{L}$.

The coupling loss factor computed with Eq. (36) is added to that of the air cavity in the SEA system. In Fig. 18 the sound reduction index of the same example described in Section 3.2.2 is shown, both for the simple leaf-cavity-leaf system and for the same wall with four studs inside. Two types of studs are considered: on the one hand a conventional S-section stud and on the other hand an acoustic stud (LR), with the shapes shown in Fig. 19. The dimensions of the studs are $d 1=70 \mathrm{~mm}, d 2=40 \mathrm{~mm}$, $d 3=10 \mathrm{~mm}, d 4=14 \mathrm{~mm}, d 5=14 \mathrm{~mm}$ and $d 6=28 \mathrm{~mm}$.

For the highest frequencies the increment in the sound transmission due to the extra path added by the studs is negligible. This happens because the transmission through the air cavity for these frequencies is already large compared to the one added by the studs. For the mid-frequency range, however, the presence of the studs reduces the sound insulation of the double wall. The improvement of the performance with the acoustic stud in front of the conventional one happens, as expected, for the mid and high frequencies. As explained in [24], the increase of flexibility due to stud shape is more relevant around the eigenfrequencies where the central part of the stud acts as a spring. These eigenfrequencies do not happen in the low-frequency range.

\section{Conclusions}

This paper has shown different aspects to take into account for the CLF estimation with numerical simulations, as well as other considerations related to the use of statistical energy analysis. They are summarised here:

- The way of computing the CLF once the deterministic problem is solved is very important for performing a good estimation. The values from which the CLF is computed already have a certain error, and therefore a study of the error propagation is required. Expressions where two similar quantities are subtracted must be avoided.

- The CLF estimated from systems consisting of only two subsystems can be used for solving larger problems with SEA. However, the CLF estimation must be performed with the same type of excitation of the problem where it will be applied. 
- A numerical estimation of the coupling loss factor between the two leaves of a double wall, allows to take into account both the resonant and the non-resonant transmission. It also allows to detect that the two analytical approaches, associated to the treatment of the cavity as a subsystem or as a connection respectively, only provide a good model of the real behaviour if they are added.

- The effect of studs can be easily considered with SEA if they are treated as line springs with frequency-dependent stiffness. The coupling loss factor associated to the studs does not always involve a significant increment of the sound transmission. The effect depends on the frequency, the type of stud and the cavity properties. The use of acoustic studs improves the insulation of the double wall in front of the conventional ones for mid and high frequencies.

\section{A Global analysis of the error propagation}

A more complete error propagation analysis is performed here, where every quantity has been considered susceptible to have errors. The analysis has been performed for the three expressions proposed in Section 2.1.

First expression:

$$
\eta_{12}=\frac{\Pi_{1, \text { in }} / \omega-\eta_{11}\left\langle E_{1}\right\rangle}{\left\langle E_{1}\right\rangle-\frac{n_{1}}{n_{2}}\left\langle E_{2}\right\rangle}
$$

The relative error in this case is

$$
\begin{aligned}
r_{\eta_{12}}=\frac{\Pi_{1, \text { in }} / \omega}{\Pi_{1, \text { in }} / \omega-\eta_{11}\left\langle E_{1}\right\rangle} & r_{\Pi_{1, \text { in }}}-\frac{\eta_{11}\left\langle E_{1}\right\rangle}{\Pi_{1, \text { in }} / \omega-\eta_{11}\left\langle E_{1}\right\rangle}\left(r_{\eta_{11}}+r_{1}\right)- \\
& -\frac{\left\langle E_{1}\right\rangle}{\left\langle E_{1}\right\rangle-\frac{n_{1}}{n_{2}}\left\langle E_{2}\right\rangle} r_{1}+\frac{\frac{n_{1}}{n_{2}}\left\langle E_{2}\right\rangle}{\left\langle E_{1}\right\rangle-\frac{n_{1}}{n_{2}}\left\langle E_{2}\right\rangle}\left(r_{n_{1}}-r_{n_{2}}+r_{2}\right) .
\end{aligned}
$$

If the coupling is weak and $\left\langle E_{1}\right\rangle / n_{1} \gg\left\langle E_{2}\right\rangle / n_{2}$, then $\Pi_{1, \text { in }} / \omega-\eta_{11}\left\langle E_{1}\right\rangle$ tends to zero and therefore

$$
r_{\eta_{12}} \simeq F_{1} r_{\Pi_{1, \mathrm{in}}}-F_{2}\left(r_{\eta_{11}}+r_{1}\right)-r_{1}
$$

where the factors $F_{1}, F_{2} \gg 1$.

Second expression:

$$
\eta_{12}=\frac{\eta_{22}\left\langle E_{2}\right\rangle}{\left\langle E_{1}\right\rangle-\frac{n_{1}}{n_{2}}\left\langle E_{2}\right\rangle}
$$

The relative error is

$$
r_{\eta_{12}}=r_{\eta_{22}}+r_{2}-\frac{\left\langle E_{1}\right\rangle}{\left\langle E_{1}\right\rangle-\frac{n_{1}}{n_{2}}\left\langle E_{2}\right\rangle} r_{1}+\frac{\frac{n_{1}}{n_{2}}\left\langle E_{2}\right\rangle}{\left\langle E_{1}\right\rangle-\frac{n_{1}}{n_{2}}\left\langle E_{2}\right\rangle}\left(r_{n_{1}}+r_{2}-r_{n_{2}}\right) .
$$

If the coupling is weak and $\left\langle E_{1}\right\rangle / n_{1} \gg\left\langle E_{2}\right\rangle / n_{2}$,

$$
r_{\eta_{12}} \simeq r_{\eta_{22}}+r_{2}-r_{1}
$$


Third expression:

$$
\eta_{12}=\frac{\Pi_{12} / \omega}{\left\langle E_{1}\right\rangle-\frac{n_{1}}{n_{2}}\left\langle E_{2}\right\rangle}
$$

The relative error is

$$
r_{\eta_{12}}=r_{\Pi_{12}}-\frac{\left\langle E_{1}\right\rangle}{\left\langle E_{1}\right\rangle-\frac{n_{1}}{n_{2}}\left\langle E_{2}\right\rangle} r_{1}+\frac{\frac{n_{1}}{n_{2}}\left\langle E_{2}\right\rangle}{\left\langle E_{1}\right\rangle-\frac{n_{1}}{n_{2}}\left\langle E_{2}\right\rangle}\left(r_{n_{1}}+r_{2}-r_{n_{2}}\right) .
$$

If the coupling is weak and $\left\langle E_{1}\right\rangle / n_{1} \gg\left\langle E_{2}\right\rangle / n_{2}$,

$$
r_{\eta_{12}} \simeq r_{\Pi_{12}}-r_{1}
$$

If the coupling is weak, the conclusion is the same as with the simplified analysis: both the second and the third expressions allow to control the error in the CLF by controlling the error of the other variables involved in the computation. However, the first expression amplifies the errors of the computed (or measured) variables, leading to a much larger error for the CLF.

\section{B CLF estimation in quasi-SEA problems}

Certain vibroacoustic systems cannot be considered as proper SEA systems, because they do not fulfill all the SEA hypotheses [18, 19]. In these quasi-SEA problems, sometimes indirect coupling loss factors must be added. Numerical simulations can also be used to estimate these factors, as shown here for the simplified example of Fig. 20. It consists of three connected subsystems, where two of them are equal.

The power balances of the three subsystems when only the first one is excited are

$$
\begin{aligned}
\Pi_{1, \mathrm{in}} / \omega & =\left(\eta_{A}^{\mathrm{int}}+\eta_{A B}+\eta_{A A}\right) E_{1}-\eta_{B A} E_{2}-\eta_{A A} E_{3} \\
0 & =\left(\eta_{B}^{\mathrm{int}}+2 \eta_{B A}\right) E_{2}-\eta_{A B} E_{1}-\eta_{A B} E_{3} \\
0 & =\left(\eta_{A}^{\mathrm{int}}+\eta_{A B}+\eta_{A A}\right) E_{3}-\eta_{B A} E_{2}-\eta_{A A} E_{1},
\end{aligned}
$$

where $\eta_{A B}$ and $\eta_{A B}$ are the coupling loss factors shown in Fig. 20, $\eta_{A A}$ is the indirect coupling loss factor and $\eta_{A}^{\text {int }}$ and $\eta_{B}^{\text {int }}$ are the internal loss factors of subsystems type A and type $\mathrm{B}$ respectively. In these balances $E_{i}$ stands for $\left\langle E_{i}\right\rangle$ as an abuse of notation aimed to simplify the formulation.

The three expressions in Eq. (47) are linearly dependent. Therefore, to compute the three unknowns $\eta_{A B}, \eta_{B A}$ and $\eta_{A A}$ another expression is required. For simplicity, it is assumed that the consistency relation between $\eta_{A B}$ and $\eta_{B A}$ holds. With this extra information, Eq. (47) becomes

$$
\begin{aligned}
\Pi_{1, \mathrm{in}} / \omega & =\left(\eta_{A}^{\mathrm{int}}+\eta_{A B}+\eta_{A A}\right) E_{1}-\eta_{A B} E_{2} n_{A} / n_{B}-\eta_{A A} E_{3} \\
0 & =\left(\eta_{B}^{\mathrm{int}}+2 \eta_{A B} n_{A} / n_{B}\right) E_{2}-\eta_{A B} E_{1}-\eta_{A B} E_{3} \\
0 & =\left(\eta_{A}^{\mathrm{int}}+\eta_{A B}+\eta_{A A}\right) E_{3}-\eta_{A B} E_{2} n_{A} / n_{B}-\eta_{A A} E_{1} .
\end{aligned}
$$


From the power balance of the second subsystem (second line in Eq. (50)), the value of $\eta_{A B}$ can be isolated as

$$
\eta_{A B}=\frac{\eta_{B}^{\mathrm{int}} E_{2}}{E_{1}+E_{3}-2 \widetilde{E}_{2}}
$$

where $\widetilde{E}_{2}=E_{2} n_{A} / n_{B}$. This expression is only dangerous in terms of error propagation if $E_{1}+E_{3} \simeq 2 \widetilde{E}_{2}$. However, this case has little physical meaning. It will only take place if the decomposition into subsystems is not properly done, leading to equal values of the modal energy at every subsystem.

The estimation of $\eta_{A A}$ can be done with the power balance of the first or the third subsystem. Using the first subsystem, it reads

$$
\eta_{A A}=\frac{\left(E_{3}-\widetilde{E}_{2}\right)\left(\Pi_{1, \mathrm{in}} / \omega-\eta_{A}^{\mathrm{int}} E_{1}\right)+\eta_{A}^{\mathrm{int}} E_{3}\left(E_{1}-\widetilde{E}_{2}\right)}{\left(E_{1}-E_{3}\right)\left(E_{1}+E_{3}-2 \widetilde{E}_{2}\right)}
$$

The expression derived from the power balance of the third subsystem is

$$
\eta_{A A}=\frac{\left(E_{3}-\widetilde{E}_{2}\right)\left(\eta_{B}^{\mathrm{int}} E_{2}+\eta_{A}^{\mathrm{int}} E_{3}\right)+\eta_{A}^{\mathrm{int}} E_{3}\left(E_{1}-\widetilde{E}_{2}\right)}{\left(E_{1}-E_{3}\right)\left(E_{1}+E_{3}-2 \widetilde{E}_{2}\right)} .
$$

From the analyses performed in Section 2.2 , it is known that $\left(\eta_{B}^{\text {int }} E_{2}+\eta_{A}^{\text {int }} E_{3}\right)$ is a more reliable expression than $\left(\Pi_{1, \text { in }} / \omega-\eta_{A}^{\text {int }} E_{1}\right)$. Therefore, it would be better to estimate $\eta_{A A}$ with Eq. (55) than with Eq. (54).

In Eq. (50), it is assumed that the consistency relation holds for the sake of simplicity. However, if this assumption is not true, another simulation (exciting a different subsystem) should be performed to add the required extra information to the problem.

\section{Acknowledgements}

The financial support of the Ministerio de Educación y Ciencia (FPU scholarship program) and the Col-legi d'Enginyers de Camins, Canals i Ports is gratefully acknowledged.

\section{References}

[1] D.A. Bies and S. Hamid. In situ determination of loss and coupling loss factors by the power injection method. Journal of Sound and Vibration, 70(2):187-204, 1980 .

[2] A. Brekke. Calculation methods for the transmission loss of single, double and triple partitions. Applied Acoustics, 14(3):225-240, 1981. 
[3] B.A. Campolina, N. Atalla, N. Dauchez, and P. Neple. Four-pole modelling of vibration isolators: Application to SEA of aircraft double-wall panels subjected to mechanical excitation. Noise Control Engineering Journal, 60(2):158-170, 2012.

[4] R.J.M. Craik. Sound transmission through buildings using statistical energy analysis. Gower Publishing Ltd., 1996.

[5] R.J.M. Craik. Non-resonant sound transmission through double walls using statistical energy analysis. Applied Acoustics, 64(3):325-341, 2003.

[6] R.J.M. Craik and R.S. Smith. Sound transmission through double leaf lightweight partitions part I: airborne sound. Applied Acoustics, 61(2):223-245, 2000.

[7] L. Cremer, M. Heckel, and E.E. Ungar. Structure-borne sound. Springer-Verlag, 1973.

[8] C. Díaz-Cereceda, J. Hetherington, J. Poblet-Puig, and A. Rodríguez-Ferran. A deterministic model of impact noise transmission through structural connections based on modal analysis. Journal of Sound and Vibration, 330(12):2801-2817, 2011.

[9] C. Díaz-Cereceda, J. Poblet-Puig, and A. Rodríguez-Ferran. The finite layer method for modelling the sound transmission through double walls. Journal of Sound and Vibration, 331(22):4884-4900, 2012.

[10] C.R. Fredö. A SEA-like approach for the derivation of energy flow coefficients with a finite element model. Journal of Sound and Vibration, 199(4):645-666, 1997.

[11] L. Gagliardini, J. Roland, and J.L. Guyader. The use of a functional basis to calculate acoustic transmission between rooms. Journal of Sound and Vibration, 145(3):457-478, 1991.

[12] P. Gelat and N. Lalor. The role and experimental determination of equivalent mass in complex SEA models. Journal of Sound and Vibration, 255(1):97-110, 2002.

[13] P. Henrici. Elements of Numerical Analysis. Wiley, New York, 1964.

[14] N.J. Higham. Accuracy and stability of numerical algorithms. Siam, 2002.

[15] C. Hopkins. Sound insulation. Elsevier Ltd., 2007.

[16] K. De Langhe and P. Sas. Statistical analysis of the power injection method. The Journal of the Acoustical Society of America, 100(1):294-303, 1996.

[17] R.H. Lyon. Statistical Energy Analysis of Dynamical Systems. M.I.T. Press, 1975 . 
[18] B.R. Mace. Statistical energy analysis, energy distribution models and system modes. Journal of Sound and Vibration, 264(2):391-409, 2003.

[19] B.R. Mace. Statistical energy analysis: coupling loss factors, indirect coupling and system modes. Journal of Sound and Vibration, 279(1):141-170, 2005.

[20] G. Maidanik. Response of ribbed panels to reverberant acoustic fields. The Journal of the Acoustical Society of America, 34(6):809-826, 1962.

[21] L. Maxit and J.L. Guyader. Estimation of SEA coupling loss factors using a dual formulation and FEM modal information, part I: theory. Journal of Sound and Vibration, 239(5):907-930, 2001.

[22] L. Maxit and J.L. Guyader. Extension of SEA model to subsystems with nonuniform modal energy distribution. Journal of Sound and Vibration, 265(2):337$358,2003$.

[23] R. Panneton and N. Atalla. Numerical prediction of sound transmission through finite multilayer systems with poroelastic materials. The Journal of the Acoustical Society of America, 100(1):346-354, 1996.

[24] J. Poblet-Puig, A. Rodríguez-Ferran, C. Guigou-Carter, and M. Villot. The role of studs in the sound transmission of double walls. Acta Acustica united with Acustica, 95(3):555-567, 2009.

[25] K. Renji and M. Mahalakshmi. High frequency vibration energy transfer in a system of three plates connected at discrete points using statistical energy analysis. Journal of Sound and Vibration, 296(3):539-553, 2006.

[26] G. Semprini and L. Barbaresi. Acoustical proprieties of light brick walls and its effects on flanking transmission. The Journal of the Acoustical Society of America, 123(5):3763, 2008.

[27] B.H. Sharp. Prediction methods for the sound transmission of building elements. Noise Control Engineering, 11(2):53-63, 1978.

[28] C. Simmons. Structure-borne sound transmission through plate junctions and estimates of SEA coupling loss factors using the finite element method. Journal of Sound and Vibration, 144(2):215-227, 1991.

[29] J.A. Steel and R.J.M. Craik. Statistical energy analysis of structure-borne sound transmission by finite element methods. Journal of Sound and Vibration, 178(4):553-561, 1994.

[30] A.N. Thite and B.R. Mace. Robust estimation of coupling loss factors from finite element analysis. Journal of Sound and Vibration, 303(3-5):814-831, 2007.

[31] N. Totaro, C. Dodard, and J.-L. Guyader. SEA coupling loss factors of complex vibro-acoustic systems. Journal of Vibration and Acoustics, 131(4):041009, 2009.

[32] T.E. Vigran. Building acoustics. Taylor and Francis, 2008. 
[33] H. Yan, A. Parrett, and W. Nack. Statistical energy analysis by finite elements for middle frequency vibration. Finite elements in analysis and design, 35(4):297$304,2000$. 


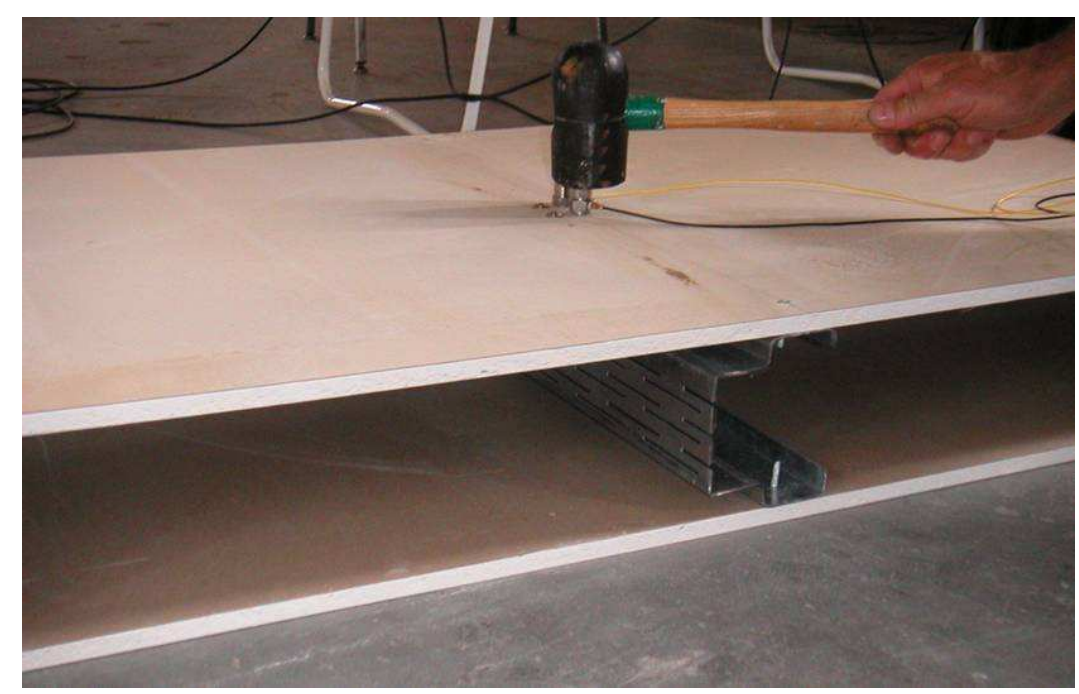

Figure 16: Picture of the location of a stud between the leaves [24].

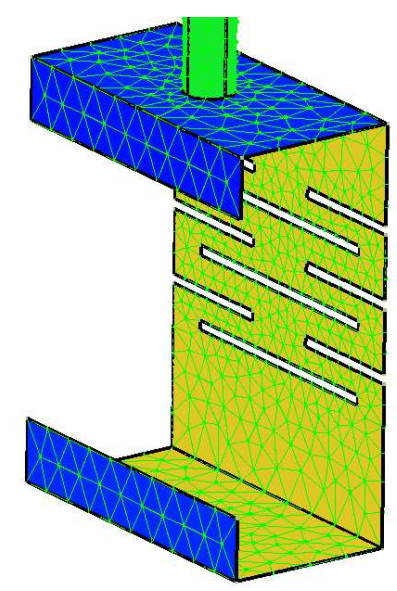

Figure 17: Finite element mesh of a stud. 


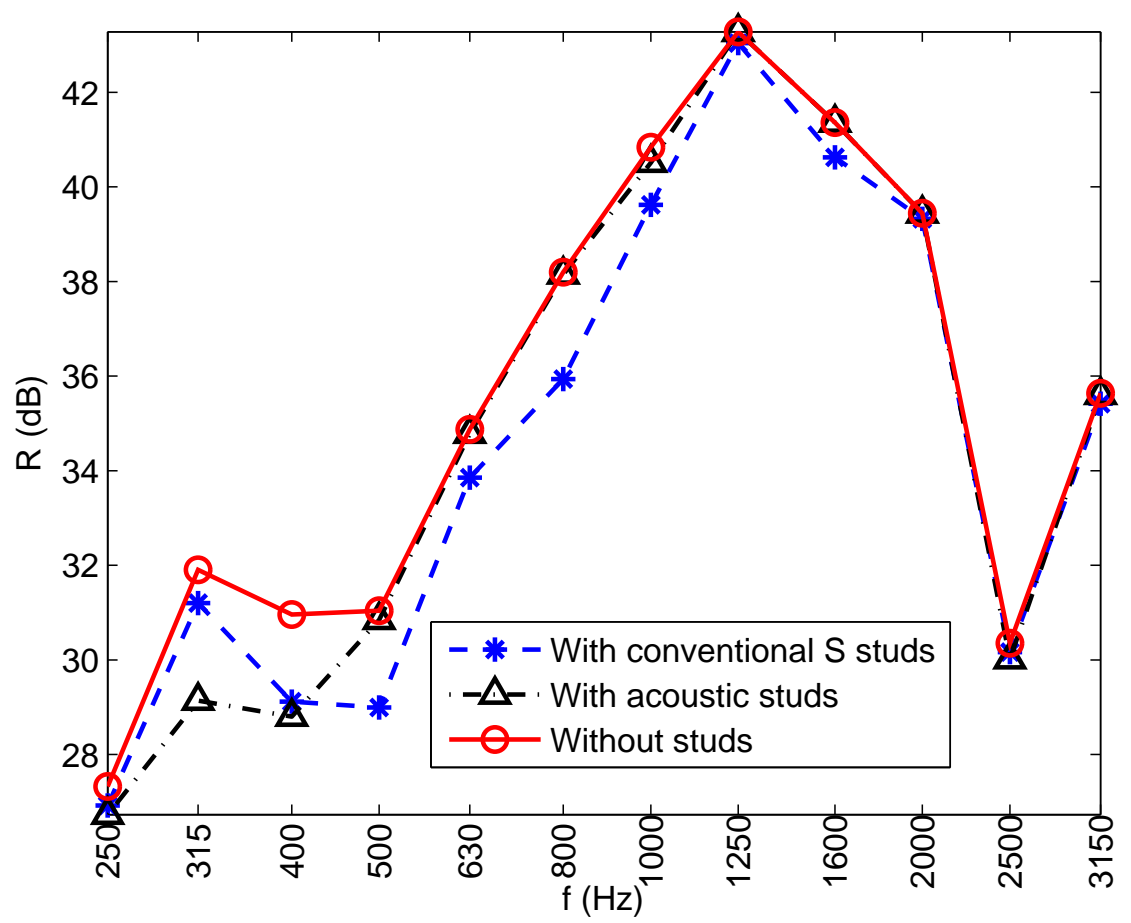

Figure 18: Effect of the studs in the sound reduction index through the double wall.

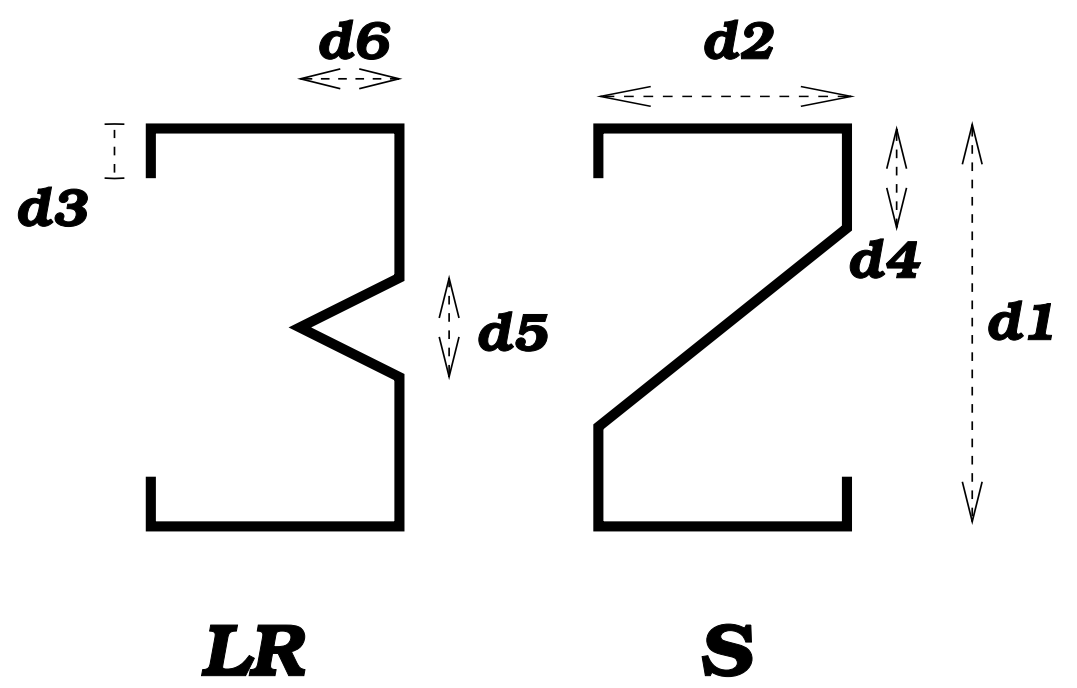

Figure 19: Section of the studs. 




Figure 20: Sketch of the quasi-SEA problem. 